\title{
The Analysis of Impact Damage under Emergency Landing Due to Airworthiness Requirements for Airplane Seat/Restraint System and Occupant

\author{
Xiao-Yan DANG ${ }^{1}$, Jin-Feng LV ${ }^{1}$
}

${ }^{1}$ AVIC, the First Aircraft Institute, Shaanxi Xi'an, 710089, China

Keywords: Crash scenatios, Crashworthiness, Pam crash, Airplane seat.

\begin{abstract}
To protect occupant under emergency landing, the seat/restraint system of airplane must be designed to has crash-resistant ability. The dynamic simulation can get the damage and impact response characteristics. The results can be helpful to modify the seat/restraint system and seat dynamic test.
\end{abstract}

\section{Introduction}

Based on the CCAR23.562, seats and the restraint of the currency aircraft must be designed to protect passengers when the aircraft is under emergency landing. But the amount of the passengers' seats is designed by testing to verify the airworthiness. The dynamic test can check up the damage of the structure and occupant, at the same time it means that it will pay out more time to complete tests. The dynamic simulation is the fast way to get test data, and is very important and useful to help designing.

Based on the CCAR23.562, seats and the restraint of the currency aircraft must be designed to protect passengers when the aircraft is under emergency landing. But the amount of the passengers' seats is designed by testing to verify the airworthiness. The dynamic test can check up the damage of the structure and occupant, at the same time it means that it will pay out more time to complete tests. The dynamic simulation is the fast way to get test data, and is very important and useful to help designing.

\section{Items of Airworthiness}

CCAR23.562 gives the rule of damage: the biggest structure load should be smaller than the broken load, and the deformation of structure can't cumber the space of saving passage, at last the restraint must be on the right location of the body.

\section{Rules of the Seat/ Restraint on Designing}

The request about seat/ restraint when the aircraft is under Emergency landing in CCAR23.562 (a):

(a) The seat and restraint system must be designed as prescribed in this paragraph to protect occupant under emergency landing:

(1) Use seats, safety belts and shoulder by the requirement;

(2)Forced the occupant on the load.

\section{Test Requests of the Seat/ Restraint}

In CCAR23.562 (b) there is a particular illuminate about dynamic tests of the seat/ restraint, the occupant simulated by a 170-pound $(77 \cdot \mathrm{kg}$ ) anthropomorphic (ATD) which have a graceful carriage.

(1) The first test, a change velocity $(\Delta \mathrm{v})$ is must larger than 31 feet per second $(9.4 \mathrm{~m} / \mathrm{s})$, and the axis of the seat/ restraint is the aircraft coordinate system. The horizontal level of the aircraft noseup pitch 60 degrees relative to the impact direction and without deflexion. The biggest deceleration which loaded on the front seat and restraint must occur in not more than 0.05 seconds after impact and reach a minimum of $19 \mathrm{~g}$. For the other seats and restraints, the biggest deceleration must occur in not more than 0.06 seconds after impact and reach a minimum of $15 \mathrm{~g}$. 
(2)The second test, a change velocity, $(\Delta \mathrm{v})$ is must larger than 42 feet per second $(12.8 \mathrm{~m} / \mathrm{s})$, and the axis of the seat/ restraint is the aircraft coordinate system. The vertical symmetry plane of the aircraft deflexion 10 degrees relative to the impact direction and without pitching, and, the biggest load is on the biggest load of the restraint. The biggest deceleration which loaded on the front seat and restraint must occur in not more than 0.05 seconds after impact and reach a minimum of $26 \mathrm{~g}$. For the other seats and restraints, the biggest deceleration must occur in not more than 0.06 seconds after impact and reach a minimum of $21 \mathrm{~g}$.

(3)Before (2), the joints or the orbit on the floor which is connected with the structure from seats and constraint must be deflexion at least 10 degree (not parallel with pitching), and make the joints or the orbit on the floor deflection 10 degree by foreordain test.

\section{Test Criterion of the Seat/Restraint}

(a) When the aircraft is under Emergency landing by CCAR23.562(c) the dynamic test result about seat/ restraint need to verify:

(1) The seat/restraint must remain hold on the anthropomorphic (ATD), although the structure may have foreseeable yielded, stretch and damage;

(2) The joints between seat/restraint and test clamp must be keep connecting, although the seat structure may have yielded;

(3) Every safe belt must be keep on the shoulder in impact;

(4) Every safe belt must be keep on the pelvis in impact;

(5) The result of the dynamic test must be verified that the passengers' heads have not heavy damage;

If occupant are likely to contact the nearby seat, structure and the other things, the restraint must be protected passengers without serious head injury so that the Head Injury Criterion (HIC) is less than 1000 units.

The value of HIC is defined:

$$
H I C=\left\{\left(t_{2}-t_{1}\right)\left[\frac{1}{\left(t_{2}-t_{1}\right)} \int_{t_{1}}^{t_{2}} a(t) d t\right]^{2.5}\right\}_{\operatorname{Max}}
$$

Where

$t_{1}$ is the initial integration time(s);

$t_{2}$ is the final integration time(s);

$t_{2}-t_{1}$ is the time of the impact on the head(s);

$a(t)$ is the total acceleration at the head gravity(in units of gravity $(\mathrm{g})$ ).

Head Injury Criterion (HIC) must be fulfilled by the test in sub-paragraph 1.2 ; otherwise using test or simulation to verify HIC.

(6) Loads on the one side shoulder is not exceed $7790 \mathrm{~N}$ (793.8kg, 7750 pounds). If using dual straps, the total strap tension loads is not exceed $8900 \mathrm{~N}(907.2 \mathrm{~kg}, 2000$ pounds).

\section{Analysis Method}

The software PAM-CRASH which based on Explicit FEM solver method. The key of the calculation is the contacts among anthropomorphic, safe belt and structures for impact problem.

\section{Simulation Theory of Contact/Impact}

FEM modal of two impact things should be constructed alone in the simulation method of contactimpact, then get the impact load by displacement condition and momentum function. Nowadays, there is four ways to solve the contact:

(a) penalty function method

Penalty function method get the stiffness by designing the contact of elements, or get to calculate the contact stiffness by the dimension and stiffness of impact structures, then get the 
contact force by multiply contact stiffness by contact displacement. The mean of physics is that there is a spring between the slave node and the penetrated through the surface, and it can limit the penetration by slave node to the penetrated through surface. This method has a deficiency, the results of contact force, contact velocity and contact acceleration has a range which the range lies on the parameter of the penalty function. But by reducing the step time can limit the range.

(b) Lagrange gene method

Contact force of the function is unknown in this method, and then resolve the function by changing the Lagrange gene and contact gene step by step, and finally it need to satisfy the condition between contact and displacement. This method increases the freedom of the system, and the condition of displacement is in equation, and it conduces many difficulties for this reason.

(c) enlarge Lagrange iterative method

The two above methods mixed can receive this method, and the core of which is penalty function method.

(d) dual tone structure analysis method based on the variation principle of condition

In this method the increment is changed with displacement and increasing displacement, then substitute variation equation with the displacement condition on the surface to set up the impact modal. Thus the modal can answered for the condition of forces, displacement, and the rule of friction. The total theories are well-rounded. But sometimes the function cannot be well solved because of the intricate iteration with many different situations in the period of calculating incensement. The principle of the penalty function method is very simple. And sand-glass is not often appeared, at the same time there is no simulation noise, and it is very accurate about conservation of momentum.

\section{Contact Surface and the Built-Off Condition}

In this phase the contact between the things of $\mathrm{A}$ and $\mathrm{B}$ is concerned. $V_{A}$ and $V_{B}$ are the configurations in initial time, and $A_{A}$ and $A_{B}$ are the borderline, and $V_{C}=A_{A} \cap A_{B}$ is the contact, and see it in the follow figure.

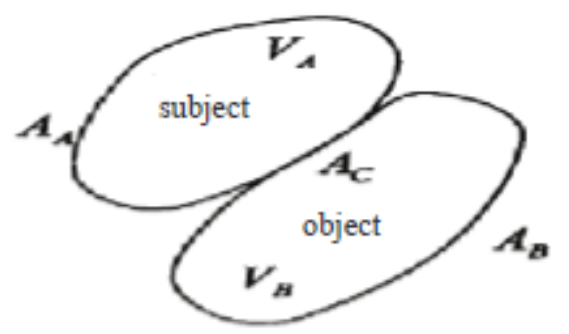

Figure 1. Two Contact each other things

Here suppose $\mathrm{A}$ is the master thing, and the oscillatory surface of $\mathrm{A}$ is the initiative surface; $\mathrm{B}$ is the slave thing, and the oscillatory surface of $B$ is the driven surface. the built-off condition can be described by the follow function:

$$
V_{A} \cap V_{B}=0
$$

In this function, it can be proved that the thing of A cannot be lapped over the thing of $\mathrm{B}$. At the beginning time we cannot know the contact node between $\mathrm{A}$ and $\mathrm{B}$, so the built-off condition is unable to described by the algebra function or the differential coefficient function of the displacement in the large deformation problem. In calculating it is necessary to contrast the coordinate value or the velocity of A and B at every step, then it can be achieved the condition of displacement:

$$
\begin{aligned}
U_{N}^{A}-U_{N}^{B} & =\left(u^{A}-u^{B}\right) n^{A} \leq\left.\mathrm{O}\right|_{A C} \\
V_{N}^{A}-V_{N}^{B} & =\left(v^{A}-v^{B}\right) n^{A} \leq\left.\mathrm{O}\right|_{A C}
\end{aligned}
$$

Where subscript $\mathrm{N}$ is the normal of contact. 


\section{The Condition of the Interface Force}

Force on interface is received by the law of action and reaction:

$$
\begin{aligned}
& t_{N}^{A}+t_{N}^{B}=0 \\
& t_{T}^{A}+t_{T}^{B}=0
\end{aligned}
$$

Where:

$t_{N}^{A}$ and $t_{N}^{B}$ are the normal force on interface of $\mathrm{A}$ and $\mathrm{B} ; t_{T}^{A}$ and $t_{T}^{B}$ are the tangential force on interface of $\mathrm{A}$ and $\mathrm{B}$ (friction force).

\section{Analysis Method of the Contact-Impact}

There is many way s about Contact force on interface by FEM, penalty function method is compared with other methods which has very well stabilization, adaptability. Here is the process about symmetrical penalty function method:

a) see figure 2 , it is necessary to concern the nearly master node ${ }^{m}$ for the anyone slave node ${ }^{n_{s}}$; check all of the master surface which is dependent with the master node ${ }^{m}$, then make sure the contact surface which could be penetrated by the slave node $n_{s}$.if the master node $m_{s}$ is not super posited with the slave node $n_{s}$ which is contacted with the master surface ${ }^{S_{i}}$ when function (3) is meted

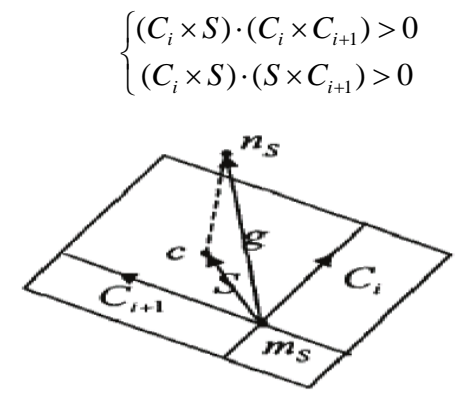

Figure 2. The contact of the slave node and the master surface

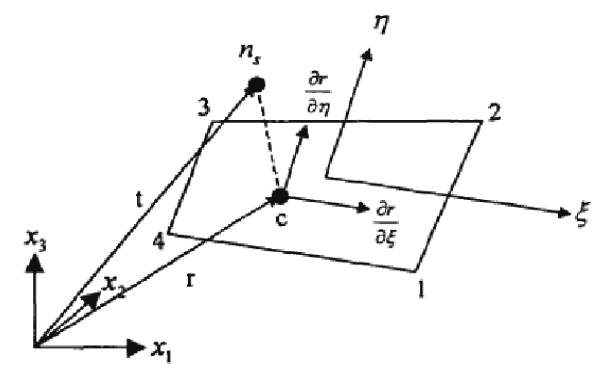

Figure 3. The relation of the slave node and the master surface

Where

$C_{i}$ and $C_{i+1}$ are the vector line on the master surface ${ }^{m_{s}} ; g$ is the vector from the master node $m_{s}$ to the slave node ${ }^{n_{s}}$; vector $S$ is the $g$,s projection on the master surface,

$$
S=g-(g \cdot m) m ; m=\left(C_{i} \times C_{i+1}\right) /\left|C_{i} \times C_{i+1}\right|
$$

If $n_{s}$ is nearly by or posited on the line of two contact surfaces, the function above may be not sure, then $S=\max \left(g \cdot C_{i} /\left|C_{i}\right|\right) \quad i=1,2, \ldots$

b) Check the contact position of the slave node ${ }^{n_{s}}$ on the master surface; 
c) The vector of any node on the master surface can be described

$$
r=f_{1}(\xi, \eta) \cdot i_{1}+f_{2}(\xi, \eta) \cdot i_{2}+f_{3}(\xi, \eta) \cdot i_{3^{\prime}}
$$

Where

$$
\begin{aligned}
& f_{1}(\xi, \eta)=\sum_{j=1}^{4} \phi_{j}(\xi, \eta) x_{i}^{j} \\
& \phi_{j}(\xi, \eta)=\frac{1}{4}\left(1+\xi_{j} \xi\right)\left(1+\eta_{j} \eta\right)
\end{aligned}
$$

$x_{i}^{j}$ is the value of the ${ }^{x_{i}}$ coordinate on the node $\mathrm{j},{ }_{1}, i_{2}, i_{3}$ is the unit vector of $x_{1}, x_{2}, x_{3}$.the position of the contact point can be solved from the following function

$$
\left\{\begin{array}{l}
\frac{\partial r}{\partial \xi}\left(\xi_{c}, \eta_{c}\right) \cdot\left[t-r\left(\xi_{c}, \eta_{c}\right)\right]=0 \\
\frac{\partial r}{\partial \eta}\left(\xi_{c}, \eta_{c}\right) \cdot\left[t-r\left(\xi_{c}, \eta_{c}\right)\right]=0
\end{array}\right.
$$

Check up the penetration.

If $l=n_{i} \cdot\left[t-r\left(\xi_{c}, \eta_{c}\right)\right]<0$, it means that the slave node $n_{s}$ has penetrated through the master surface $^{c\left(\xi_{c}, \eta_{c}\right)}$, and the contact node lies on the master surface. $n_{i}$ is the unit vector of outside normal at the contact node of the master surface.

$$
n_{\varepsilon}=\frac{\partial r}{\partial \xi}\left(\xi_{t}, \eta_{t}\right) \times \frac{\partial r}{\partial \eta}\left(\xi_{\epsilon}, \eta_{t}\right) /\left|\frac{\partial r}{\partial \xi}\left(\xi_{\epsilon}, \eta_{t}\right) \times \frac{\partial r}{\partial \eta}\left(\xi_{t}, \eta_{t}\right)\right|
$$

If $1 \geq 0$, it means that the slave node $n_{s}$ has not penetrated through the master surface, i.e. two things has not appeared impact/contact, then straightly search the next ${ }^{n+1}$.

e) if the slave node has penetrated through the master surface, then it appeared the normal contact force $f_{s}$ between the slave node $n_{s}$ and the contact node c

$$
f_{s}=-l k_{t} n_{t}
$$

Where

$k_{t}$ is the stiffness gene on the master surface, then

$$
k_{i}=\left\{\begin{array}{lll}
f k_{i} A_{i}^{2} / V_{i} & \text { solid } & \text { element } \\
f k_{i} A_{i} / L_{i} & \text { plate } & \text { element }
\end{array}\right.
$$

$k_{i}$ is bulk modulus of the contact element; $A_{i}$ is the area of master element; ${ }_{i}$ is the maximum diagonal length of the plate element; $f$ is the scale gene of the contact stiffness, the default value is 0.10 . if the penetrability value is much larger, it needs to zoom in the penalty function gene, but if $f>0.4$ then the calculation is not stability, unless reduce the step time.

Force vector $f_{s}$ on interface is acted at the slave node $n_{s}$ by the law of action and reaction, then there is a counter force ${ }^{-f_{s}}$ at the contact node $\mathrm{C}$ on the master surface. The next step is equivalently distributed the contact force at the node $\mathrm{C}$ to the node of the master element:

$$
f_{j m}=-\phi\left(\xi_{c}, \eta_{c}\right) f_{s} ; j=1,2,3,4 ;
$$


$\phi_{j}\left(\xi_{c}, \eta_{c}\right)$ is the 2D shape function on the master surface, and at the contact node C: $\sum_{j=1}^{4} \phi_{j}\left(\xi_{c}, \eta_{c}\right)=1$

f) Tangential contact force (friction force)

If the normal contact force is $f_{s}$ at the slave node ${ }^{n_{s}}$, maximum friction force is $F_{Y}=\mu\left|f_{s}\right|, \mu$ is friction coefficient. Supposed at the last step ${ }^{t_{n}}$ the friction force is $F^{n}$ at the slave node ${ }^{n_{s}}$, then at the current step $t_{n+1}$ the potential friction force $F^{*}$ is, $F^{*}=F^{n}-k \Delta e . k$ is the interface stiffness, $\Delta e=r^{n+1}\left(\xi_{c}^{n+1}, \eta_{c}^{n+1}\right)-r^{n+1}\left(\xi_{c}^{n}, \eta_{c}^{n}\right)$.At the current time the friction force can got by the following function:

$$
F^{n+1}=\left\{\begin{array}{cl}
F^{*} & \text { if }\left|F^{*}\right| \leq F_{Y} \\
F_{Y} F^{*} /\left|F^{*}\right| & \text { if }\left|F^{*}\right|>F_{Y}
\end{array}\right.
$$

Then calculate friction force at any node the by the law of action and reaction. If the static friction coefficient is $\mu_{s}$, the dynamic friction coefficient is $\mu_{d}$, it needs to smooth the function by exponential interpolation function:

$$
\mu=\mu_{d}+\left(\mu_{s}-\mu_{d}\right) e^{-C|V|}
$$

Where

$V=\Delta e / \Delta t, \Delta t$ is the step time, $\mathrm{C}$ is attenuation gene.

g) put known quantities: the contact force vector $f_{s}, f_{m}$,and the friction force vector in the load matrix, and then analysis the dynamic characteristic.

Slave nodes and master nodes are solely solved circularly by symmetrical penalty function method. only solved slave nodes circularly is 'separated and smoothed disposable arithmetic', it is mainly applied to the contact things that are rigid bodies. It can save time.

\section{The Modal}

\section{Anthropomorphic Modal}

An occupant simulated by a $77 \cdot \mathrm{kg}$ anthropomorphic (ATD) which is 50 percent Hybrid III aeronautic man-made modal. Multibody system Dynamics modal contains 17 stiffness parts and 16 joints. Every part is the corresponding body, and there is a joint between every two parts. The mass and the moment of inertia is equivalent to the part of the human body, and the figure is same too. The rotational joints between every two parts are simulated by nonlinear springs and damps. Figure 4 is the anthropomorphic modal.

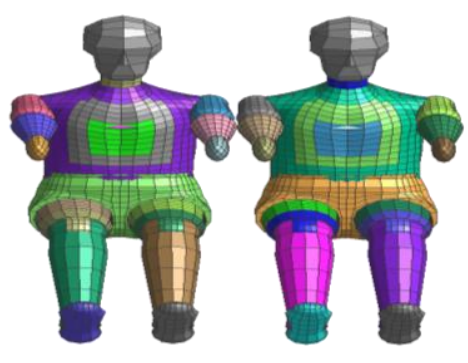

Figure 4. Anthropomorphic modal

\section{Seats and Restraint System}

There is 2 safe restraint system with 3 points at the back seats of the one aircraft, and two points fixed on the bottom of seats and that another point is on the top structure. And there is 3 NAS62044 bolts between the seat and structure. 
The safety belt is simulated by membrane element. This element can simulated the things which are thin and curving, and it has even stress distributing at the thickness direction and 0 curving stiffness. Figure 5 is the structure and safe belt modal.

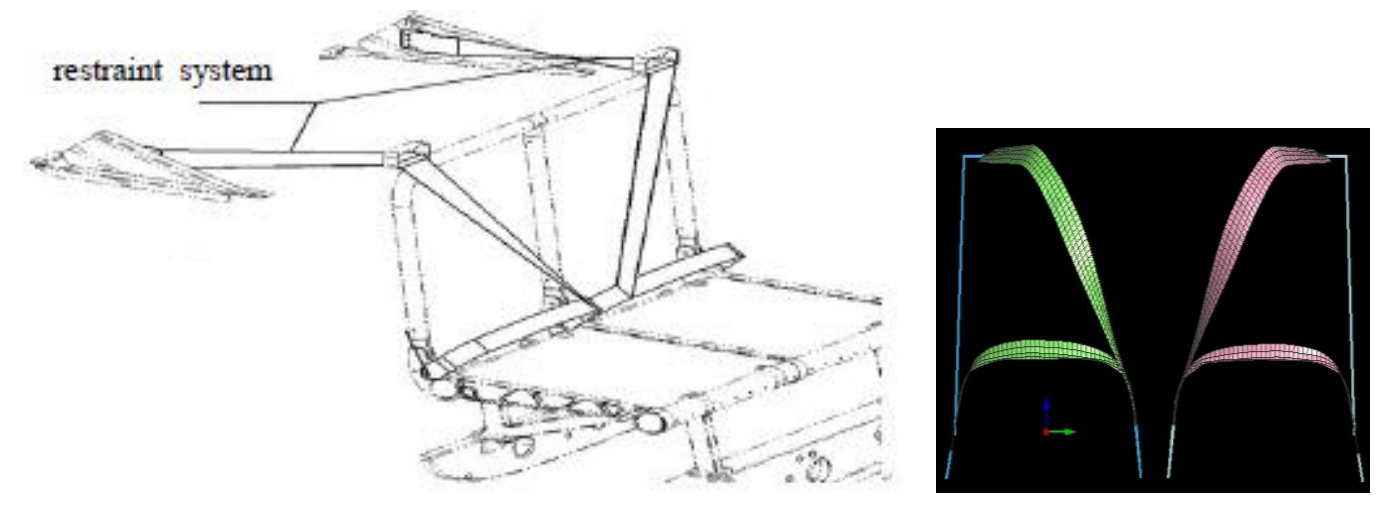

Figure 5. Seats and restraint system

\section{Result}

The maximum stress is appeared at the joint between the tubing and the side of the angle strut, and deformation has made the material into plastic area, but not separated, this result is proved that the seat can protected occupant safe.

The axial force with time is changed, and the two side maximum axial force are $8423.48 \mathrm{~N}$ and $7685.88 \mathrm{~N}$ at time $5.6 \mathrm{~ms}$, figure 6 is curves which show the force changed with time. By the airworthiness requirement, if there is one belt the load through belt must be less than $7790 \mathrm{~N}$, so the left result already has exceeded the limit value. The reason is that the modal of the belt has a little difference, and the result need to be validated with data of tests
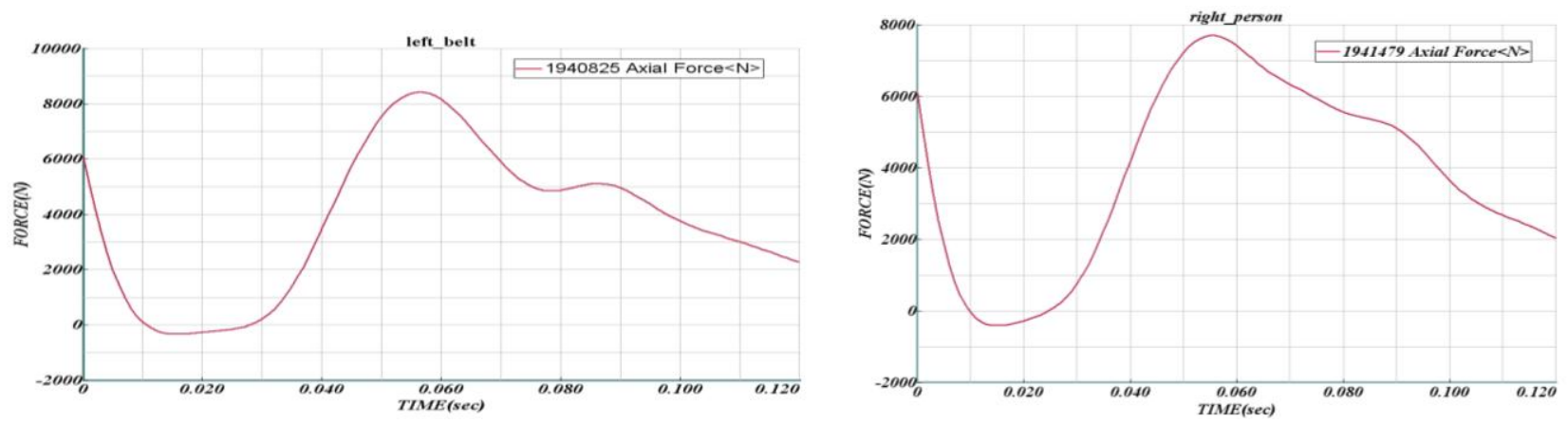

Figure 6. The axial force of left/right belt

Figure 7,8 and 9 show the curves changed that is the acceleration response with time at the different part of body, because of there is no fraise at front of the heads, so the damage is not the reality value. The maximum acceleration response $50.8 \mathrm{~g}$ is appeared at the pelvis of the right person.
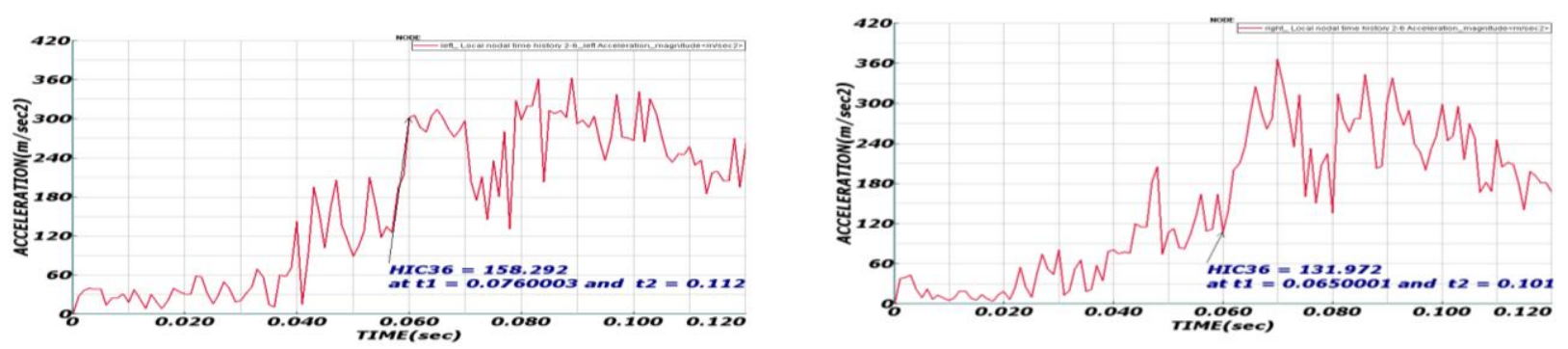

Figure 7. Acceleration response changed with time at the head of left/right person 

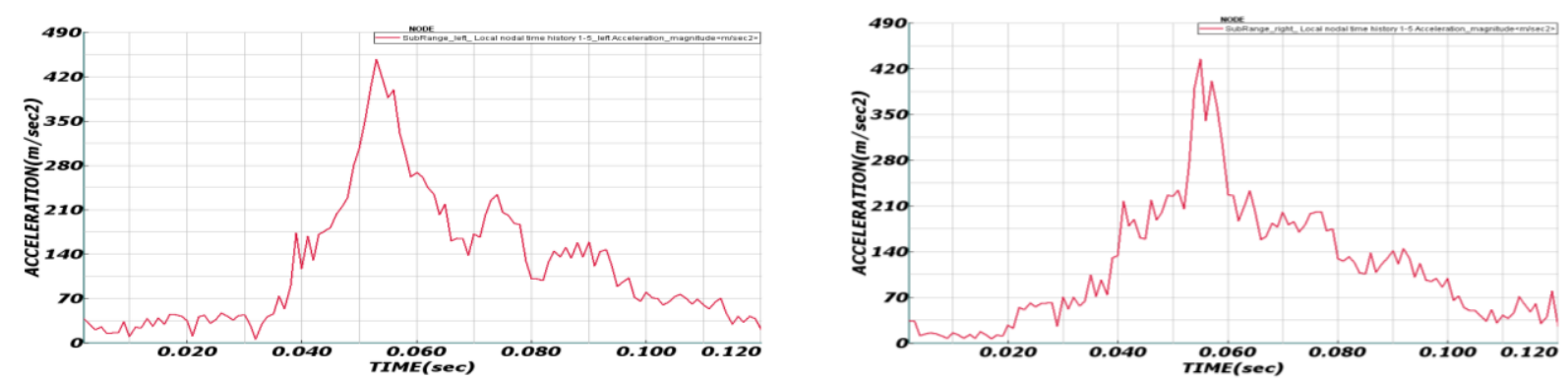

Figure 8. Acceleration response changed with time at the breast of left/right person
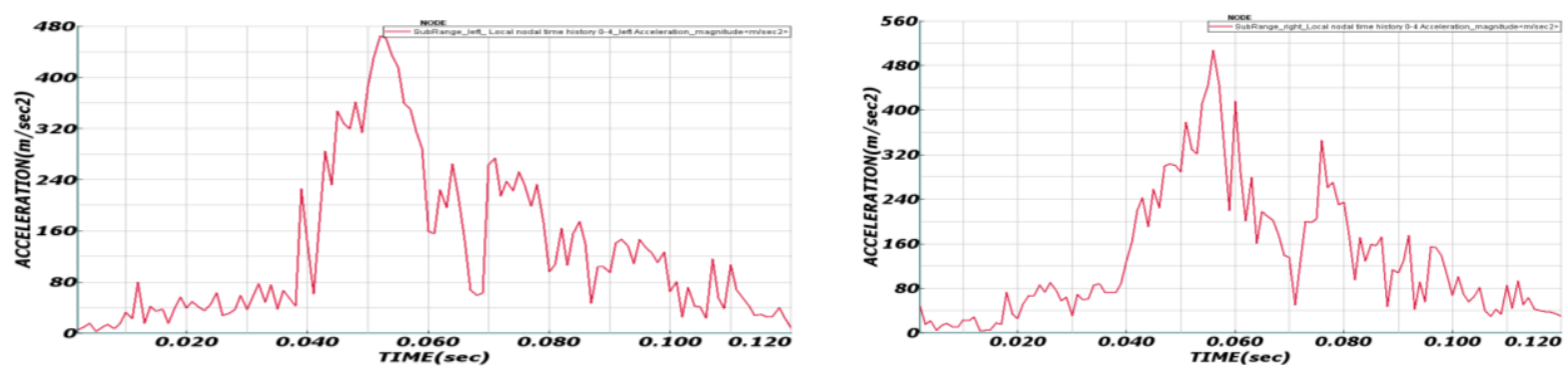

Figure 9. Acceleration response changed with time at the pelvis of left/right person

\section{Conclusion}

Based on the airworthiness CCAR23.562: Emergency landing dynamic conditions, the model of two person seats has been built and the dynamic characteristics of the seat/restraint system with occupant were acquired through dynamic simulation. The seats and restraint are safe for the passengers after impact, except a little structure deformation. Meanwhile, the safety belts, and shoulder harnesses are still fixed on the body to protect occupant.

The parameters of safety belts are not exact, so the tests are still required to verify the airworthiness requirements.

\section{Reference}

1. CCAR 23 R3 Airworthiness standards: normal, utility, acrobatic, and commuter category airplanes. (in Chinese) 